\section{An Active Investment Strategy for EMBnet - AGM workshop report, Oeiras, June 2015}

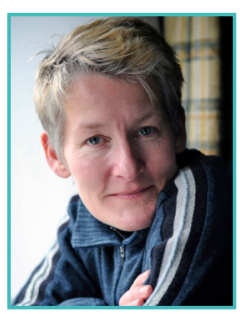

\section{Teresa K. Attwood}

University of Manchester, Manchester, United Kingdom

Received 23 March 2016; Published 1 April 2016

Attwood TK (2015) EMBnet.journal 21, e867. http://dx.doi. org/10.14806/ej.21.0.867.

\section{Introduction}

EMBnet's 2015 AGM and associated activities were hosted in Oeiras (PT), from 10 to 12 June. Marking the 27th formal meeting of EMBnet, the event was an opportunity to take stock and carefully consider EMBnet's future. Earlier in the year, the Operational Board (OB) had agreed that EMBnet would benefit from active investment in specific projects to galvanise its members and drive the community forward. Accordingly, this year, the AGM activities included a full-day $O B$ meeting as a prelude to a one-day workshop for the new strategy to be discussed and refined, followed by the traditional business meeting, where the investment plans would be presented for formal endorsement by the Board.

The purpose of this report is to set out the background to the workshop, to explain the motivation for creating the new investment strategy, to detail its principal themes, and to summarise the workshop's main conclusions.

\section{Overview of the current status}

Motivation for the investment strategy: the need for action

One year ago, we discussed some of the changes that would need to take place at the 2015 AGM. In particular, three members of the Executive Board (EB) would be obliged to step down, and a future new - yet to be identified leadership team would have to assume responsibility for taking EMBnet forward. Early planning was deemed essential to help manage this major change efficiently and effectively. In consequence, an extraordinary Face-To-Face (F2F) meeting of the $O B$ was arranged to discuss i) the location for the AGM; ii) how to manage the forthcoming leadership changes; and iii) how to re-invigorate EMBnet. Members of the OB were asked to submit written proposals in advance of the meeting, outlining future programmes of work to help address these issues.

During the meeting (which, for convenience, took place from 18-20 February 2015, at Schiphol), Pedro Fernandes volunteered to host the AGM at the Instituto Gulbenkian de Ciência, Oeiras. It was agreed to hold the meeting in June.

In terms of EMBnet's leadership changes, it was agreed that a call for new EB candidates should be made as soon as possible, including a deadline for response. It was recognised, however, that this call might not be successful in eliciting candidates; in the worst-case scenario, it might also prove difficult to find volunteers during the AGM itself. Regardless of the outcome, given the scale of the change (i.e., the simultaneous loss of the Chair, Secretary and Treasurer), the $O B$ recommended that, during the AGM, an Interim Board (IB) should be put in place to mentor any new EB candidates, to oversee implementation of the new investment strategy, and to help evaluate its success at the 2016 AGM.

The final component of this extraordinary $O B$ meeting was a lengthy and vigorous debate around each of the submitted proposals for rejuvenating EMBnet. All agreed that a significant investment should be made to stimulate focused activities at the heart of EMBnet. Two main

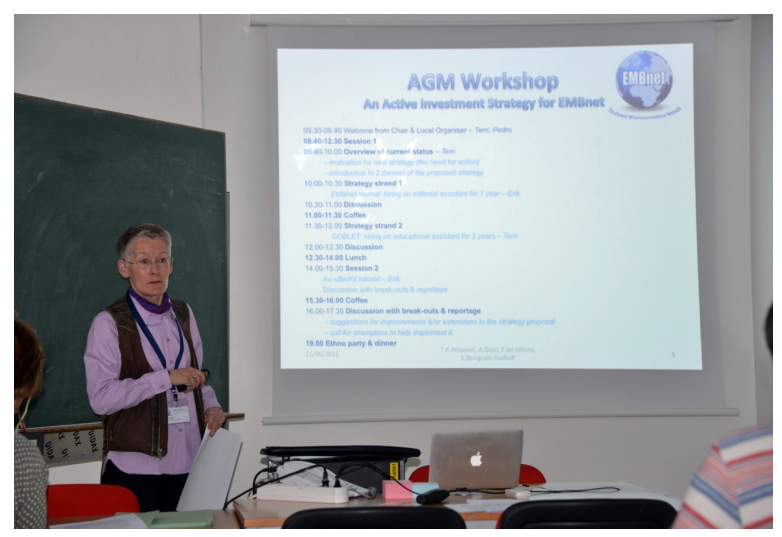

Figure 1. Terri Attwood presents the agenda of the EMBnet AGM 2015 workshop. 
themes eventually emerged as candidates for funding: a) supporting EMBnet.journal; and b) strengthening the link with GOBLET.

After the meeting, a Virtual General Meeting (VGM) was called to share and discuss this outcome; however, aside from the $\mathrm{OB}$ itself, only three members of EMBnet attended. The 2015 AGM workshop was therefore an important opportunity for wider discussion, to solicit feedback on each theme of the strategy (including suggestions for improvements, extensions and/or other modifications), and to commit to implementing them.

Introducing theme 1: supporting EMBnet.journal

The leap from EMBnet.news to EMBnet.journal had been enormous, and managing the change had been challenging. Journal production is almost entirely dependent on Lubos Klucar; some members of the Executive Editorial Board also give support with reviewing, copyediting, writing editorials, etc. The number of articles is also a concern, but until the Journal has acquired an impact factor, attracting articles is likely to remain problematic.

The proposed solution was to hire an Editorial Assistant for one year to help with journal publicity, solicitation of new articles, gaining sponsors, and so on. The idea would be to closely monitor progress, and to evaluate the impact at the next AGM: on this basis, the decision would finally be taken whether to continue producing the journal, or perhaps to revert back to EMBnet.news (EMBnet's formerly successful newsletter and progenitor of the journal).

Introducing theme 2: strengthening the link with GOBLET

As part of the 2012 AGM, EMBnet had invited leaders of nine other organisations to a workshop to discuss global challenges in bioinformatics training. Each of these organisations had some sort of Education and Training (E\&T) initiative, each with similar aims, and each with the same problem: how to deliver tangible benefits to their communities with limited funds and just a handful of time-pressed volunteers. The outcome of that meeting was an agreement to create a Global Organisation for Bioinformatics Learning, Education and Training - GOBLET.

Six months later, following the model of EMBnet, GOBLET was formally established as a
Stichting. The GOBLET Foundation uses a similar fee structure to EMBnet, but with two more tiers: for organisations, the tiers are bronze (€250), silver (€500), gold $(€ 1,000)$ and platinum $(€ 2,500)$. EMBnet is a Gold Member.

Three years on, with $\sim 40$ organisational and individual members, GOBLET is one of the most significant, most visible and most successful of EMBnet's recent initiatives: it is supported by publications; it is promoted at ISMB; it is recognised worldwide; it is also recognised as a partner of ELIXIR. Having spear-headed the formation of GOBLET, there is a golden opportunity here for EMBnet to make an even stronger statement, to strengthen our commitment to GOBLET.

The proposal was to make a ring-fenced donation to GOBLET in order to hire an assistant for two years, to professionally develop branded training materials, to disseminate these via GOBLET's Training Portal, to advertise them on EMBnet's website, and publish them in EMBnet. journal, where appropriate. As with the Editorial Assistant, the idea would be to monitor progress closely, and evaluate the impact of this dedicated role at the next AGM.

\section{The Investment Strategy}

\section{Supporting EMBnet.journal}

The set-up supporting the operation of EMBnet. journal is complicated. The technical infrastructure - the server - is hosted in Sweden; the administration is performed in Slovakia. Day-to-day reviewing, copy-editing, editorial writing, and so on, is done by members of the full Editorial Board, some of whom also endeavour to promote the journal. Overall, this process runs fairly smoothly, if rather slowly at times.

One of the main impediments to the success of the journal is that it lacks an Impact Factor (IF), which inhibits researchers from submitting their articles. To move forward, the journal needs to be indexed in PubMed. Of course, this is not all - all members of EMBnet can help by submitting articles to the journal, and encouraging others to do so. But this is not enough: ideally, for the journal to operate on a more professional footing, it needs the support of an assistant to help with:

- email correspondence;

- announcements via different media;

- advertisement (both acquiring advertisers and promoting the journal); and 
- creating Special Editions (inviting guest editors in specific subjects/areas).

It had therefore been proposed to hire an assistant for one year to help with such routine tasks and to implement new promotional strategies, to improve and support the production of the journal: $€ 1,000$ per month would be available to support this post. The successful candidate would be expected to work closely with the journal's Executive Editorial Board to prioritise the tasks, to report on a monthly basis to the EB, and to report in person at the $A G M$, where the impact of the work would be evaluated. Here, measures of success might include:

- tripling the number of peer-reviewed articles published;

- tripling the readership (measured in different ways); and

- attracting new income from advertisements.

It was recognised that $€ 1,000$ per month posed a possible threat to the project, as it might be insufficient to attract candidates of a suitable calibre. However, despite the acknowledged risk, the proposal was considered essential to help progress the journal. Overall, then, this component of the investment strategy was supported by all workshop participants.

\section{Strengthening the link with GOBLET}

EMBnet was created $\sim 27$ years ago as a government-mandated, government-supported infrastructure to provide 'bioinformatics' services to European national communities - in particular, to distribute the EMBL Data Library (this was before the term bioinformatics had been coined in this field, and hence EMBnet was the European Molecular Biology network not the European Bioinformatics network). However, with the advent of easy-to-use browsers, which revolutionised access to the Internet, the world changed, and this once-essential service role of EMBnet was largely superseded. Eventually, EMBL Bank and its associated services found a sustainable home at the European Bioinformatics Institute (EBI), and a much larger initiative was ultimately created, not just to sustain EMBL Bank, but to support all EBI core resources and services - this was ELIXIR, a new, more ambitious, more substantially funded sort of 'EMBnet', based on the principle of nationally-funded Nodes mandated to provide bioinformatics services to their communities.
Despite these radical changes in the European bioinformatics landscape, EMBnet continued to function - often, arguably, without much real sense of purpose. The last major funding that EMBnet received for its activities was via the European project, EMBCORE. However, this was at a time when EMBnet had expanded its borders to include a range of developing countries. These could not benefit from European grants, thereby creating an awkward division in EMBnet's funding stream that was hard to manage. Since then, no funds (from Europe or elsewhere) have been sought to unify all EMBnet members around a common project.

Many former members of EMBnet have since found a new focus in ELIXIR, and several current members now play active roles there. Some of those have been particularly active in the realms of bioinformatics E\&T - indeed, it was partly because of this that EMBnet took the lead in creating GOBLET, as this was an opportunity to put EMBnet's E\&T activities on the map globally. With this in mind, in 2014, EMBnet's Publicity and Public Relations (P\&PR) Project Committee (PC) undertook a survey to try to gain a view of what members felt were EMBnet's core values. The survey identified various strong points: these included EMBnet.journal; EMBnet.digest; EMBnet QuickGuides; E\&T activities (GOBLET); its worldwide network (albeit not including North America or Japan); the network's expertise; and its Linkedln Group (which provides greater visibility). On the flip side, the survey also highlighted that EMBnet is weak in terms of research and new tool innovation, that it offers few services online (given the number of groups the network encompasses), that there are few interactions between members outside AGMs, and there is no common project to unify members.

Overall, the survey concluded that EMBnet's major core values are in bioinformatics E\&T, capacity building and networking. To build on its strengths, it was recommended that modern E\&T initiatives should be developed, reinforcing our alliances with other societies and networks that are proficient in this area, and that new initiatives should be launched, including projects to galvanise new activities and recruit younger members. Some of the recommendations were very specific and gave considerable food for thought. In particular, Laurent Falquet suggested that "we should orient ourselves towards a bioinformat- 
ics education community, by publishing more QuickGuides, online courses, and perhaps focus EMBnet.journal on publishing more educationrelated articles", that EMBnet "should drop the old trend of providing cutting-edge services because we are not strong in research anymore", and finally, that EMBnet should "become one of the most important GOBLET members, by contributing as much as possible." He argued that "Providing well-maintained services useful for teaching purposes would be a definitive strength (e.g., a Galaxy/Chipster server, with specific small databases and well-documented training exercises.)"

Shortly after this rather provocative survey outcome, the 2014 AGM took place in Lyon. This event was notable for a number of reasons, not least because EMBnet's Secretary provided a critical review of EMBnet, concluding that active steps need to be taken to help drive EMBnet forward (Attwood TK, 2014).

During the $\mathrm{OB}$ meeting, taking into account the survey results, and reflecting on the AGM critique, a strong recommendation was made to review EMBnet's position with respect to E\&T. Overall, it was suggested to refocus EMBnet's activities and, as part of a new investment strategy, to make a public move to become a 'life-time' member of GOBLET, making a substantial donation - €25K - to match. Such a move would allow an E\&T assistant to be hired for two years, and would have a number of clear benefits. Specifically, it would:

- highlight EMBnet's commitment to E\&T;

- provide opportunities to create high-quality, jointly-branded materials (tutorials, exercises, manuals, updated/revitalised/professionalised QuickGuides, etc.) for advertisement on EMBnet's website, publication, where appropriate, through EMBnet.journal and dissemination via GOBLET's Training Portal;

- help to focus EMBnet's work and demonstrate a clear vision and purpose;

- allow concentration on the core values identified by the survey -bioinformatics E\&T, capacity building and networking - but in partnership with a burgeoning and active training organisation;

- allow the EMBnet brand to persist through GOBLET;
- encourage more EMBnet members to engage with GOBLET.

The candidate would be expected to prioritise a set of tasks (working closely with relevant groups in GOBLET and EMBnet), to report monthly to the organisations' respective EBs, and to report in person at their AGMs. As with the Editorial Assistant, however, it was recognised that €1,000 per month might not attract candidates of a suitable calibre. Although acknowledged to be risky, particularly in terms of the size of the investment, the proposal was nevertheless considered strategically important for EMBnet, and highly valuable for GOBLET. Consequently, there was warm support from all workshop participants.

\section{eBioKit Tutorial}

Erik presented the latest version of the eBiokit, describing its content, functionality and usefulness, not just for teaching activities but also for research purposes in countries where Internet connections are either very slow or absent. He also described how the Kit is distributed, upgraded and updated, and presented courses and past experiences that have demonstrated its efficacy. Workshop participants were invited to use the eBioKit, to suggest further improvements, and to collaborate on the implementation of additional tools, courses and resources that are not currently present within the system.

\section{Discussion and conclusions}

The final session of the workshop had been intended to allow a more general discussion of the investment strategy and to solicit possible improvements, extensions and/or additions to its two main strands. However, as both had been enthusiastically endorsed earlier in the workshop, further discussion was unfruitful.

\section{EMBnet Fellowships}

Moving on, the workshop focused on the final component of the overall investment plan. During the 2014 AGM, a new 'Fellowship' scheme had been approved as a possible method for getting new blood into EMBnet. At that time, it had been agreed that two Fellowships, each amounting to $€ 2,000$, would be awarded during 2014-2015 initially, the term was set at 18 months, but this was subsequently revised to 1-year terms. For a variety of reasons, however, the initiative had not progressed. The workshop participants were therefore invited to revisit the Fellowship scheme, 
and to suggest possible EMBnet-related projects that could be advertised on the website. Possible activities included:

- development of a QuickGuide app for mobile devices;

- development of a camera-ready template for QuickGuides;

- website development, including new contribute content, RSS feeds, etc:;

- development of a Utopia Documents plugin for EMBnet.journal;

- development of training materials for the eBioKit; and

- creating a Linux-based eBioKit VM.

It was once more agreed that the Fellowship scheme should proceed.

\section{Re-design of EMBnet.journal}

An additional project discussed during this session concerned the need to professionalise the look-and-feel of EMBnet.journal. This had originally been considered a potential Fellowship project. However, it was recognised that this work is intimately tied up with the success of strand 1 of the investment strategy - hence, there would be little point hiring an assistant to help invigor- ate and promote the journal, if the journal's style was not re-worked at the same time. Further, this task could not sensibly be part of the Editorial Assistant's role, because the skill-sets required for these positions are quite different. It was generally felt that this was both important and urgent, and that $€ 2 \mathrm{~K}$ should be made available specifically to engage an individual to re-design the journal pages. The workshop participants agreed that this was a sensible way forward.

\section{Communications and Community}

During the general discussions, a proposal was made to automate the process by which EMBnet collects and disseminates news. While EMBnet. digest has attempted to fill the 'news' gap created by the transition of EMBnet.news to EMBnet. journal, the digest is time-consuming to produce, and selective in content (this is deliberate, as the digest is intended to reflect news from the EMBnet community rather than generic bioinformatics information that pervades the Internet). However, there are tools available that facilitate more automated approaches for scraping and collating news from the Internet - e.g., Scoop. it and Paper.li. Together, such tools could be

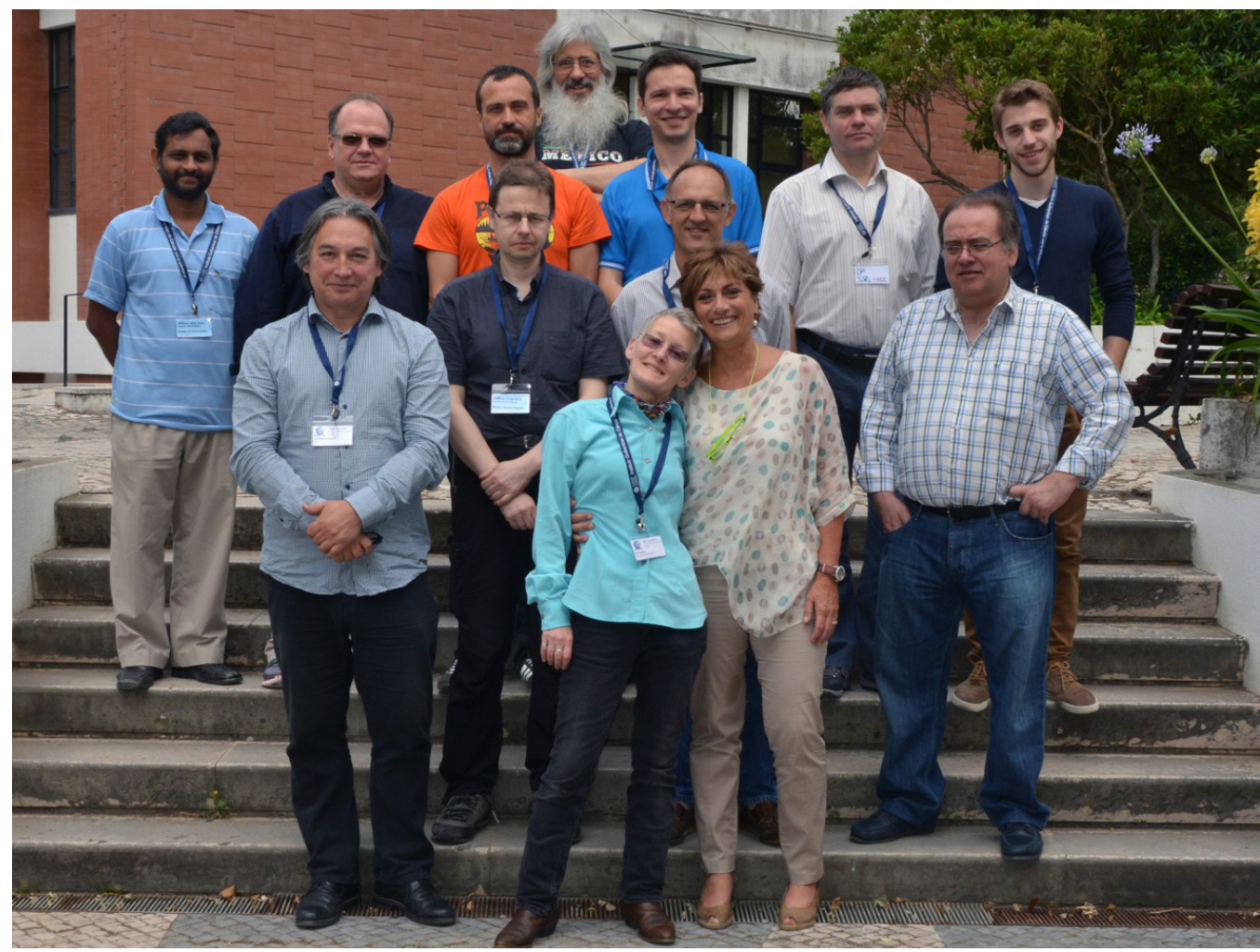

Figure 2. Group picture of the EMBnet 2015 AGM workshop participants, Oeiras (PT). 
used to provide an alternative, more 'lightweight' method of news generation, creating a kind of 'new look' EMBnet.digest. It was agreed that, with Pedro's guidance, Axel Thieffry would look into this more closely.

\section{IT for Life Scientists}

George Magklaras mentioned that he had been contacted by members of USENIX (the Advanced Computing Systems Association/ USENIX is a brand name in the field of computing professionals) to organise a conference track dedicated to life-science computing needs, focusing especially on how to train IT professionals to cater for life scientists. It had been proposed to arrange a call with them (including himself, Pedro and Terri Attwood), within the next couple of weeks. George was convinced that organising conferences with other bodies to educate IT professionals about the IT needs of life scientists was valuable for EMBnet to do on behalf of the community, and hence he would follow this up and set a date for the meeting.
Another idea that emerged from this general discussion was that EMBnet could publish screencasts. Given the right subject, these can be very popular, they can help drive traffic to websites, and are relatively straightforward to produce. As a 'proof of concept', George suggested that he could create some short screen-casts, for example, to supplement existing QuickGuides.

\section{Close of meeting}

This had been a very constructive and productive workshop. Terri thanked all for their participation, for their suggestions of new activities and for their positive support of the investment proposals, which would be taken forward for endorsement at the business meeting the next day; with that, the meeting closed.

\section{References}

Attwood TK (2014) EMBnet, the Global Bioinformatics Network: a report on the workshop and 26th AGM, Lyon, May 2014. EMBnet.journal 20, e786. nttp:///dx.doi.org/10.14806/ ej. 20.0 .786 